\title{
Robotic assistance in urological microsurgery: initial report of a successful in-vivo robot-assisted vasovasostomy
}

\author{
G. De Naeyer $\cdot$ P. Van Migem $\cdot$ P. Schatteman $\cdot$ \\ P. Carpentier $\cdot$ E. Fonteyne $\cdot$ A. Mottrie
}

Received: 9 April 2007 / Accepted: 29 April 2007 / Published online: 30 May 2007

(C) Springer London 2007

\section{Introduction}

During recent years many urologists have been convinced of the advantages of robot-assisted, minimally invasive urological procedures.

Because microscope-assisted vasovasostomy is a rather uncommon procedure, most urologists lack microsurgical experience. Use of the da Vinci robotic system (Intuitive Surgical, Mountain View, CA, USA) virtually eliminates the microsurgical challenge of tremor, limited dexterity, and miniaturized instrumentation.

Different centres have investigated the use of robotic assistance in vasovasal anastomosis in animal and human ex-vivo models. According to the current literature we are the first European centre to report a successful in-vivo robot-assisted vasovasostomy using the da Vinci robotic system.

\section{Case report}

A 34-year-old man desiring to restore fertility after a previous vasectomy was scheduled for vasovasostomy. On the basis of previous experience from robot-assisted radical prostatectomies we decided to perform this operation with technical assistance from the da Vinci robot instead of the microscope.

After dissecting and preparing both vasal ends in the conventional manner, the robot was positioned as a continuation

G. De Naeyer $(\bowtie) \cdot$ P. Van Migem $\cdot$ P. Schatteman

P. Carpentier · E. Fonteyne - A. Mottrie

Department of Urology, Onze-Lieve-Vrouw Hospital,

Moorselbaan 164, 9300 Aalst, Belgium

e-mail: geertdenaeyer@yahoo.com of the operating table at the feet of the patient. The $0^{\circ}$ camera was centred over the scrotum and the two robotic arms were loaded with microsurgical robotic needle holders. The vasovasal anastomosis was performed using a one-layer technique with a single armed 8-0 Prolene suture on a $6.5 \mathrm{~mm}$ long $3 / 8$-circle needle. Motion scaling was set at a ratio of $5: 1$.

Six equally spaced full-thickness sutures were placed in a circumferential pattern. The three posterior sutures were tied sequentially, and all three anterior sutures were placed before they were tied. All sutures were tied with six throws. The threads were cut by a table assistant.

After closing the scrotal wound in two layers the patient was transferred to the recovery room.

\section{Results}

The total operating time was $120 \mathrm{~min}$; of this the robotic assistance time was approximately $80 \mathrm{~min}$. There were no intra-operative problems; in particular there were no loose stitches, bent needles, broken sutures, or crash injuries of the vasal stumps.

Bed rest was regarded as imperative for the first $72 \mathrm{~h}$ immediately post-operative. There were no post-operative complications. Semen analysis after 3 months showed $120 \times 10^{6}$ viable spermatozoa $\mathrm{mL}^{-1}$, which confirms a patent anastomosis.

\section{Discussion}

The da Vinci robotic system was introduced to urology approximately seven years ago to enable more precise tissue dissection and to help increase the feasibility of 
laparoscopic radical prostatectomy [1]. Nowadays the da Vinci robot is used for a wider variety of procedures [2], and because of its enhanced dexterity and its superior visualisation we believe its role now can be expanded even further to include microsurgery in urology. Robot-assisted microsurgical feasibility studies have even been performed for corneal surgery [3], glottic surgery [4], and plastic surgery when performing microvasular anastomoses [5]. Because urological microsurgical procedures remain difficult to perform, especially for less-trained urologists, we found it an opportunity to use the da Vinci robotic system for vasovasal anastomosis.

This first case report is encouraging. With a 3D view and a magnification up to 30 times visualisation of the small vasal structures posed no problem. The microsurgical needle holders were able to handle the fine needle and sutures without problems. Because motion scaling was set at the finest downscaling, detailed suture placement was performed. A major advantage of robotic assistance in microsurgery is, of course, the elimination of tremor.

In the recent literature in-vivo robot-assisted vasovasostomy or vaso-epididymostomy has only been reported by Mani Menon at the Vattikuti Urology institute at Detroit [2]. Animal and human ex-vivo studies have been performed widely. Most studies revealed patency was comparable but operating time was longer for the robot-assisted procedures $[6,7]$. One study showed that robotic vasovasostomy was significantly quicker than the conventional microsurgical technique and that patency rates were higher the robotic procedure [8].

The lack of tactile sensation or haptic feedback should not be regarded as a disadvantage - by continued practice and training the surgeon becomes aware of visual cues, for example suture stretching, and is better able to integrate such optical data, obviating the need for tactile feedback. Indeed, in the above mentioned animal and human ex-vivo studies the lack of tactile sensation or feedback did not compromise anastomotic patency.

Because vasovasostomy is a rather uncommon procedure and most urologists lack microsurgical experience, the use of the da Vinci robotic system virtually eliminates the microsurgical challenge of tremor, limited dexterity, and miniaturized instrumentation.

\section{Conclusion}

This case report shows that robotic vasovasostomy is feasible and technically possible. We believe that for less experienced microsurgeons the anastomosis can be performed with greater ease and precision than conventional microscope-assisted vasovasostomy. This supposes some experience with the robotic system, of course. We do, of course, also realise that this cannot be proven by a single case report. More formal studies must be performed to evaluate this technique further. We hope that, at least, use of the da Vinci robot in a new urological application will be stimulated by this case report.

\section{References}

1. Menon M, Tewari A, Baize B, Guillonneau B, Vallancien G (2002) Prospective comparison of radical retropubic prostatectomy and robot-assisted anatomic prostatectomy: the Vattikuti Urology Institute experience. Urology. 60(5):864-868

2. Ashokk and Mani Menon. Robotics in Urology. Curr Opin Urol 14:89-93

3. Tsirbas A, Dutson E, Mango C (2007) Robotic ocular surgery. Br J Ophthalmol. 91(1):18-21

4. O' Malley BW, Weinstein GS, Hockstein NG (2006) Transoral robotic surgery (TORS): glottic microsurgery in a canine model. J Voice 20(2):263-268

5. Katz RD, Taylor JA, Rosson GD, Brown PR, Singh NK (2006) Robotics in plastic and reconstructive surgery: use of a telemanipulator slave robot to perform microvascular anastomoses. J Reconstr Microsurg 22(1):53-57

6. Kuang W, Shin PR, Oder M, Thomas AJ Jr. (2005) Robotic-assisted vasovasostomy: a two-layer technique in an animal model. Urology. 65(4):811-814

7. Kuang W, Shin PR, Matin S, Thomas AJ Jr. (2004) Initial evaluation of robotic technology for microsurgical vasovasostomy. J Urol 171(1):300-303

8. Schiff J, Li PS Goldstein M (2004) Robotic microsurgical vasovasostomy and vasoepididymostomy: a prospective randomized study in a rat model. J Urol 171(4):1720-1725 